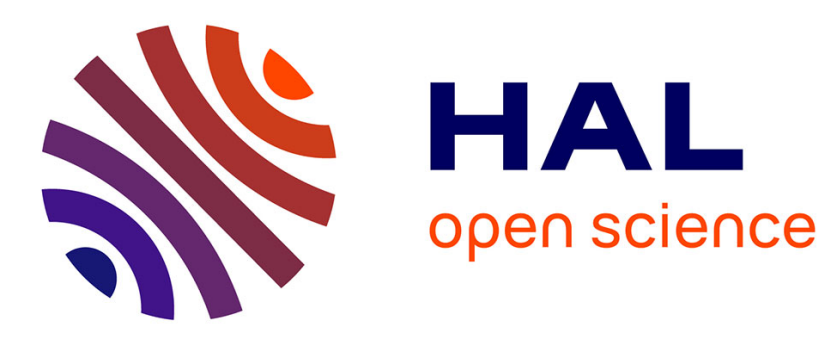

\title{
VPS51 biallelic variants cause microcephaly with brain malformations: A confirmatory report
}

Annette Uwineza, Jean-Hubert Caberg, Janvier Hitayezu, Stephane Wenric, Leon Mutesa, Yoann Vial, Séverine Drunat, Sandrine Passemard, Alain Verloes, Vincent El Ghouzzi, et al.

\section{To cite this version:}

Annette Uwineza, Jean-Hubert Caberg, Janvier Hitayezu, Stephane Wenric, Leon Mutesa, et al.. VPS51 biallelic variants cause microcephaly with brain malformations: A confirmatory report. European Journal of Medical Genetics, 2019, 62 (8), pp.103704. 10.1016/j.ejmg.2019.103704 . hal02859833

\section{HAL Id: hal-02859833 \\ https://hal.science/hal-02859833}

Submitted on 8 Jun 2020

HAL is a multi-disciplinary open access archive for the deposit and dissemination of scientific research documents, whether they are published or not. The documents may come from teaching and research institutions in France or abroad, or from public or private research centers.
L'archive ouverte pluridisciplinaire HAL, est destinée au dépôt et à la diffusion de documents scientifiques de niveau recherche, publiés ou non, émanant des établissements d'enseignement et de recherche français ou étrangers, des laboratoires publics ou privés. 


\title{
VPS51 biallelic variants cause microcephaly with brain malformations: a confirmatory report
}

\begin{abstract}
Annette Uwineza ${ }^{1}$ §, Jean-Hubert Caberg ${ }^{2}$, Janvier Hitayezuㄴ, Stephane Wenric ${ }^{3}$, Leon Mutesa ${ }^{1}$, Yoann Vial ${ }^{4,5}$, Séverine Drunat ${ }^{4,5}$, Sandrine Passemard ${ }^{4,5}$, Alain Verloes ${ }^{4,5}$, Vincent El Ghouzzi ${ }^{5}$, Vincent Bours $^{2}$
\end{abstract}

\author{
${ }^{1}$ Center for Human Genetics, College of Medicine and Health Sciences, University of Rwanda, \\ Kigali, Rwanda \\ ${ }^{2}$ Center for Human Genetics, Centre Hospitalier Universitaire, University of Liege, Liege, Belgium \\ ${ }^{3}$ GIGA-Research, Human Genetics Unit, University of Liege, Liege, Belgium; \\ ${ }^{4}$ Department of Genetics, AP HP - Robert Debré University Hospital, Paris, France. \\ ${ }^{5}$ PROTECT, INSERM UMR1141, Université de Paris, Paris, France
}

\section{§Correspondence:}

Annette Uwineza, MD, PhD

Center of Human Genetics

College of Medicine and Health Sciences

University of Rwanda, Rwanda

Tel: (+250) 788741577; email address: A.UWINEZA@ur.ac.rw 


\begin{abstract}
Genome-wide linkage analysis and whole exome sequencing undertaken in two siblings with delayed psychomotor development, absent speech, severe intellectual disability and postnatal microcephaly, revealed a homozygous intragenic deletion in VPS51, which encodes the vacuolar protein sortingassociated protein, one the four subunits of the GARP and EARP complexes that promotes the fusion of endosome-derived vesicles with the trans-Golgi network (GARP) and recycling endosomes (EARP). This observation supports a pathogenic effect of VPS51 variants, which has only been reported previously once, in a child with microcephaly. It confirms the key role of membrane trafficking in normal brain development and homeostasis.
\end{abstract}

\title{
Keywords:
}

VPS51, GARP, EARP, Golgipathies, Golgi, endosomes, postnatal microcephaly, neurodevelopmental disorders, Rwanda 


\section{Introduction}

Neurodevelopmental disorders (NDDs) encompass a wide range of intellectual, behavioral and motor disabilities (van Bokhoven, 2011). High-throughput sequencing has allowed to more readily capture variations causing subtle or atypical phenotypes, especially in NDDs (Hu et al., 2014). Recently, the role of membrane trafficking during brain development and maturation has been highlighted with a central contribution played by the Golgi apparatus and its impact on key processes : neurogenesis, neuronal migration, myelination and maturation of postmitotic neurons ((Passemard et al., 2017; Rasika et al., 2019). In particular, the Golgi-associated retrograde protein (GARP) acts in promoting the retrograde fusion of endosome-derived carriers with the trans-Golgi network (TGN) (Bonifacino and Hierro, 2011; Conibear and Stevens, 2000; Perez-Victoria et al., 2010). GARP is a multisubunit complex made of four distinct proteins (VPS51, VPS52, VPS53 and VPS54). GARP differs only in one subunit with another tethering complex, known as the endosome-associated recycling protein (EARP) complex, in which the VPS54 subunit is substituted by syndetin (VPS50). EARP ensures the fusion of endosome-derived carriers with recycling endosomes and therefore promotes recycling of endocytic membranes back to the plasma membrane (Masschaele et al., 2017; Schindler et al., 2015). Among the five subunits of the GARP/EARP complexes, VPS53 was the first to be associated with a human disease. Loss-of-function mutations in VPS53 have been associated with two forms of degenerative NDDs, autosomal recessive pontocerebellar hypoplasia type 2E (PCH2E, MIM \#615851), characterized by a severe early-onset neurodegeneration with profound intellectual disability (ID), progressive microcephaly, spasticity, and early-onset epilepsy (Feinstein et al., 2014), and progressive encephalopathy with edema, hypsarrhythmia and optic atrophy (PEHO) characterized by a severe developmental delay, limb and facial edema, intractable epilepsy, optic atrophy and dysmorphic features (Hady-Cohen et al., 2018). Recently, a 6-year-old patient with severe global developmental delay, pontocerebellar abnormalities, microcephaly, hypotonia, epilepsy and several systemic and peripheral dysfunctions has been reported (Gershlick et al., 2019). This girl was found to carry compound heterozygous variants in VPS51 affecting both GARP and EARP complexes.

Here, we report two Rwandan sisters with a homozygous three base pairs intragenic deletion in VPS51 associated with developmental delay, absent speech, severe ID and postnatal microcephaly. 
The present study confirms the key role of the GARP and EARP complexes in normal brain development and homeostasis.

\section{Patients and Methods \\ Patient 1}

Patient 1, seen in the Centre of Medical Genetics of Rwanda University, in Kigali, was born to firstcousin once removed parents of Rwandese origin $(f=1 / 32)$, for severe psychomotor developmental delay. This girl was born by normal delivery from an uneventful pregnancy (birth weight: $3600 \mathrm{~g}$ ). Head circumference was not recorded. At 1 month of age, she had a poorly described episode of fever with loss of consciousness treated by an unknown medication. Further development was severely delayed. She was able to sit unsupported at age 3 . At the first academic evaluation, at age 5 , she was still unable to walk and had no language. Clinical examination revealed microcephaly (head circumference of 46,5 cm: $-3.23 \mathrm{SD})$, a weight of $15 \mathrm{~kg}(-1.36 \mathrm{SD})$ and a height of $118 \mathrm{~cm}(+1.8 \mathrm{SD})$. She had hypertelorism, upturned nasal tip, short philtrum, thick vermilion of the upper lip, large teeth (Fig. 1 A), high arched palate, multiple dental carries, left internal strabismus, and bilateral pes planus. Brain CT-scan showed Dandy Walker variant anomaly with a cyst of the posterior fossa, communicating with the 4 th ventricle, and mild ventriculomegaly. At the age of 9 , she had ataxic gait. The language was still absent. Brain MRI showed mild vermis atrophy, a thin corpus callosum, dilated lateral ventricles and a mega cisterna magna (Fig. 2 A,B). Serum transferrin isoelectrofocusing excluded congenital disorder of glycosylation (CDG).

\section{Patient 2}

Patient 1's sister was 30-month-old at examination (Fig 1 B). She was born at term following an uneventful pregnancy and delivery (birth weight $3200 \mathrm{~g}$ ). At 12 months, she developed an episode of fever, diarrhoea and vomiting and became hypotonic. At 30 months, she was not able to walk and had no speech. Feeding difficulties at home lead to severe hypotrophy. She had upturned nasal tip and left internal strabismus (Fig $1 \mathrm{~B})$. Her head circumference was $43 \mathrm{~cm}$ (- $4.56 \mathrm{SD})$, her weight was $7 \mathrm{~kg}(<$ $-3 \mathrm{SD})$ and her height was $83 \mathrm{~cm}(-2.3 \mathrm{SD})$. Brain MRI showed no malformation of the brain but a reduced white matter volume and a thin corpus callosum with a normal vermis (Fig. 2 C, D). 


\section{Methods}

Genomic DNA was extracted from peripheral blood leukocytes using the standard phenol/chloroform method. Whole-genome SNP array was performed in patients, their parents and one unaffected sibling using HumanOmni2.5 BeadChip (Illumina, San Diego, CA). Fifty nanograms of DNA were processed according to Illumina's Infinium HD Assay Super protocol. Arrays were scanned with an iScan System and data were extracted by the Genome Studio software (Illumina, San Diego, CA) and analysed using the PLINK software. Exome sequencing (WES) was performed in both patients and their parents. Library preparation, sequencing, alignment and variant calling were performed in the Unit of Human Genetics, GIGA, at the University of Liege (Belgium). Exons were captured using the Agilent SureSelect Human All Exon V5+UTR (Agilent Technologies, Santa Clara, CA, USA), and sequence was generated on a Hiseq2000 instrument (Illumina San Diego, CA, USA). Base calling was performed using the Illumina CASAVA software. All the raw reads were aligned to the reference human genome (GRCh37 / hg19) using the Burrows-Wheeler aligner (BWA). Optical and PCR duplicates were marked and removed with Picard tools (http://picard.sourceforge.net). Single nucleotide variants and small insertions/deletions were then called using the GATK Unified Genotyper v2.7. Variant annotation was performed using ANNOVAR. Variant screening was restricted to genes included in homozygous loci revealed by homozygosity mapping. Variants with at least a minor allele frequency (MAF) of 0,01\% in dbSNP138, 1000 genomes, ExAC and ESP6500 databases were excluded. Missense variants were tested for mutational effect by prediction algorithm with Polyphen-2, Sift, Provean, mutation taster, and CADD score. The analysis process was based on the latest release of GnomAD, dbSNP, ExAC databases and the literature review. The variant identified by WES was confirmed by Sanger sequencing in the patients and their parents (primers sequence available on request). The study was approved by the Rwandan National Ethics Committee (N³94/RNEC/2013) and informed consents were obtained from the participants.

\section{Results}

Homozygosity mapping revealed a large homozygous locus on chromosome 11 (chr11:44,081,150_74,821,392) of about $30 \mathrm{Mb}(11 \mathrm{p} 11.2-\mathrm{q} 13.4)$ in both sisters. WES revealed a 
variant in the VPS51 gene (reference sequence : NM_013265.3) consisting in an in-frame deletion of a CTT codon in exon 5 (c.1419_1421del / p.(Phe474del) ) detected at the homozygous state in both sisters and at the heterozygous state in both parents and in the unaffected sister. Phe474 is a highly conserved residue. This mutation was present in only 2 individuals in the GnomAD database (MAF:0.00083\%) and was never reported at the homozygous state.

\section{Discussion}

In this study, WES was performed in two patients from a Rwandan consanguineous family with NDD. Our patients were selected from a cohort of a previously analysed series of 50 Rwandan patients presenting ID and global development delay with or without dysmorphic features (Uwineza et al., 2014). The phenotype of our patients includes minor dysmorphic features, a severe global developmental delay, a microcephaly, and a reduced volume of the subcortical white matter associated with vermis atrophy in the eldest sister.

Although the deletion identified in the present study does not induce a frameshift in the coding sequence of VPS51, the loss of the highly conserved Phe474 is predicted to impact the function and/or stability of the subunit, possibly by altering a site of interaction with other partners. Furthermore, this mutation has never been reported in patients of African origin suggesting that this mutation is not a rare polymorphism associated with an ethnic group.

A single 6-year-old patient has recently been reported with compound heterozygous mutations (c.1468C>T/p.Asp745Thrfs*93 and c.2232delC/p.Arg490Cys) in VPS51 (Gershlick et al., 2019). The patient had failure to thrive, severe ID, microcephaly, thin corpus callosum, pontocerebellar abnormalities (small vermis and thin brainstem) and a Dandy-Walker variant. EEG showed electrical status epilepticus in sleep (ESES) requiring anticonvulsant therapy. She further had cortical blindness, chronic cholestasis requiring ursodiol treatment, edema of the lower limbs reminiscent of PEHO syndrome, and some dysmorphic features (including epicanthal folds, long eyelashes, upturned nasal tip, a thin upper lip, full cheeks, increased hair on the upper back. Isoelectric focusing showed a hypoglycosylated profile of serum transferrin Authors showed that the c.2232delC produces an unstable frameshifted protein 54 aminoacids longer than the wild type and that the c.1468C $>\mathrm{T}$ 
mutation produces a stable protein that assembles less efficiently with the other GARP/EARP subunits. In skin fibroblasts from the patient, the levels of fully-assembled GARP and EARP complexes were lowered. The distribution of the cation-independent mannose 6-phosphate receptor was altered and swelling of lysosomes was noted. Our patients appear thus to show a milder phenotype lacking epilepsy, the PEHO-like features (edema, optic impairment), the liver involvement and abnormal glycosylation profile of serum transferin.

Clinical features of our patients also have similarities with those of patients reported with mutation in VPS53, such as microcephaly and developmental delay in patients presenting PCH2E (Feinstein et al., 2014). However, our patients do not have epilepsy and their developmental delay is less severe than those with PCH2E. Interestingly, mutations in both VPS53 and SEPSECS, the gene for PCH2D (OMIM \#613811), can result in PEHO-like phenotype or PCCA, suggesting that these conditions belong to a common clinical spectrum (Hady-Cohen et al., 2018). In agreement with these observations, defective Golgi proteins are increasingly linked to a wide spectrum of neurodevelopmental defects that have been recently classified as "Golgipathies" (Rasika et al., 2019) and that usually associate progressive microcephaly and ID with white matter defects.

The observation of Gershlick and colleagues (Gershlick et al., 2019) that not only the proteasomal degradation of VPS51 but also a single aminoacid substitution in its sequence can result in a decreased stability and/or efficiency of the whole GARP and EARP complexes indicates that VPS51 integrity is essential to GARP/EARP function. Of interest, the substitution reported in this paper is 16-aminoacid close to the Phenylalanine deletion observed in the present study. On the other hand, a residual activity of GARP/EARP complexes is most likely necessary to avoid embryonic lethality and may explain why these NDDs, although very severe, are viable. Further studies are obviously required to validate the consequence of this mutation on both complexes.

Among the four subunits of the complex, VPS51 is required for the proper recruitment of GARP complex at the Golgi by promoting its interaction with the SNARE machinery (Conibear et al., 2003). In EARP, VPS51 likely plays a similar function in promoting SNARE-mediated membrane fusion events. VPS51 also assembles with the other three subunits of GARP/EARP complexes in a 
stoichiometric manner which implies that its instability likely compromises that of the whole complexes (Perez-Victoria et al., 2010). Therefore, VPS51 lies at the core of GARP/EARP complexes and thereby participates to many functions essential for cell homeostasis such as traffic and recycling of endosomes, acid hydrolase sorting, lysosome function, endosomal cholesterol traffic and autophagy (Perez-Victoria et al., 2010). That the phenotype resulting from the deficiency of these complexes is mainly neurodevelopmental is somehow surprising regarding their ubiquitous expression throughout life and their critical function in maintaining cellular homeostasis. The same issue has been raised and discussed recently for a number of Golgipathies (Rasika et al., 2019). More studies are now required to understand why the nervous system appears more vulnerable than other organs to the deregulation of the endomembrane system. 


\section{Figures}

Figure 1.

Patient 1 (left) and 2 (right)

Figure 2. MRI of both siblings in T1 weight images (A,C: sagittal - B, D: axial). $A$ and $B: M R I$ of the patient 1 at age $9 ; C \& D$ MRI of the patient 2 aged 30 months. Note the thin corpus callosum and the reduced volume of white matter for both siblings. Cerebellum (vermis and lobes) shows atrophy in patient 1, whereas it is preserved in patient 2 , suggesting a progressive atrophy with age. 


\section{References}

Bonifacino, J.S., Hierro, A., 2011. Transport according to GARP: receiving retrograde cargo at the trans-Golgi network. Trends Cell Biol 21, 159-167. https://doi.org/10.1016/j.tcb.2010.11.003

Conibear, E., Cleck, J.N., Stevens, T.H., 2003. Vps51p Mediates the Association of the GARP (Vps52/53/54) Complex with the Late Golgi t-SNARE Tlg1p. Mol. Biol. Cell 14, 1610-1623. https://doi.org/10.1091/mbc.e02-10-0654

Conibear, E., Stevens, T.H., 2000. Vps52p, Vps53p, and Vps54p form a novel multisubunit complex required for protein sorting at the yeast late Golgi. Mol Biol Cell 11, 305-323.

Feinstein, M., Flusser, H., Lerman-Sagie, T., Ben-Zeev, B., Lev, D., Agamy, O., Cohen, I., Kadir, R., Sivan, S., Leshinsky-Silver, E., Markus, B., Birk, O.S., 2014. VPS53 mutations cause progressive cerebello-cerebral atrophy type 2 (PCCA2). J Med Genet 51, 303-308. https://doi.org/10.1136/jmedgenet-2013-101823

Gershlick, D.C., Ishida, M., Jones, J.R., Bellomo, A., Bonifacino, J.S., Everman, D.B., 2019. A Neurodevelopmental Disorder Caused by Mutations in the VPS51 Subunit of the GARP and EARP Complexes. Hum. Mol. Genet. https://doi.org/10.1093/hmg/ddy423

Hady-Cohen, R., Ben-Pazi, H., Adir, V., Yosovich, K., Blumkin, L., Lerman-Sagie, T., Lev, D., 2018. Progressive cerebello-cerebral atrophy and progressive encephalopathy with edema, hypsarrhythmia and optic atrophy may be allelic syndromes. Eur J Paediatr Neurol. https://doi.org/10.1016/j.ejpn.2018.07.003

Hu, W.F., Chahrour, M.H., Walsh, C.A., 2014. The diverse genetic landscape of neurodevelopmental disorders. Annu Rev Genomics Hum Genet 15, 195-213. https://doi.org/10.1146/annurevgenom-090413-025600

Masschaele, D., De Ceuninck, L., Wauman, J., Defever, D., Stenner, F., Lievens, S., Peelman, F., Tavernier, J., 2017. RNF41 interacts with the VPS52 subunit of the GARP and EARP complexes. PLoS One 12, e0178132. https://doi.org/10.1371/journal.pone.0178132

Passemard, S., Perez, F., Colin-Lemesre, E., Rasika, S., Gressens, P., El Ghouzzi, V., 2017. Golgi trafficking defects in postnatal microcephaly: The evidence for "Golgipathies.” Prog. Neurobiol. 
153, 46-63. https://doi.org/10.1016/j.pneurobio.2017.03.007

Perez-Victoria, F.J., Schindler, C., Magadan, J.G., Mardones, G.A., Delevoye, C., Romao, M., Raposo, G., Bonifacino, J.S., 2010. Ang2/fat-free is a conserved subunit of the Golgi-associated retrograde protein complex. Mol Biol Cell 21, 3386-3395. https://doi.org/10.1091/mbc.E10-050392

Rasika, S., Passemard, S., Verloes, A., Gressens, P., El Ghouzzi, V., 2019. Golgipathies in Neurodevelopment: A New View of Old Defects. Dev Neurosci in press.

Schindler, C., Chen, Y., Pu, J., Guo, X., Bonifacino, J.S., 2015. EARP is a multisubunit tethering complex involved in endocytic recycling. Nat Cell Biol 17, 639-650.

https://doi.org/10.1038/ncb3129

Uwineza, A., Caberg, J.H., Hitayezu, J., Hellin, A.C., Jamar, M., Dideberg, V., Rusingiza, E.K., Bours, V., Mutesa, L., 2014. Array-CGH analysis in Rwandan patients presenting development delay/intellectual disability with multiple congenital anomalies. BMC Med Genet 15, 79. https://doi.org/10.1186/1471-2350-15-79

van Bokhoven, H., 2011. Genetic and Epigenetic Networks in Intellectual Disabilities. Annu. Rev. Genet. 45, 81-104. https://doi.org/10.1146/annurev-genet-110410-132512 


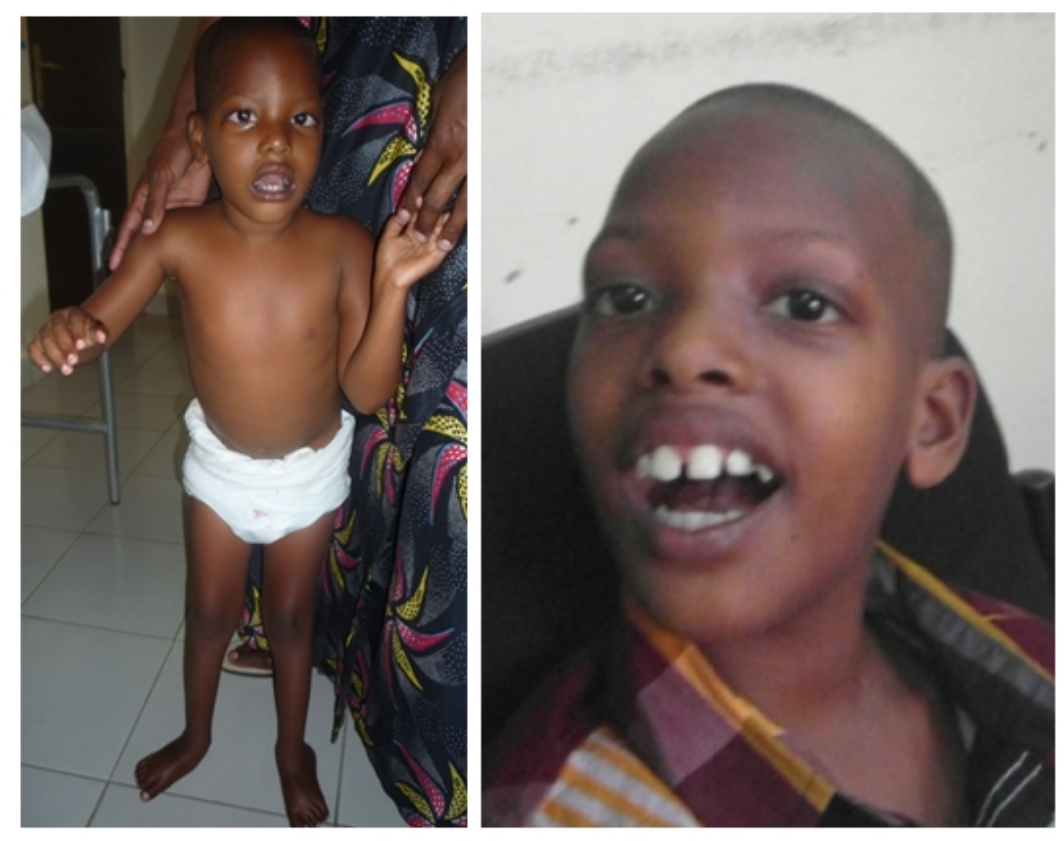




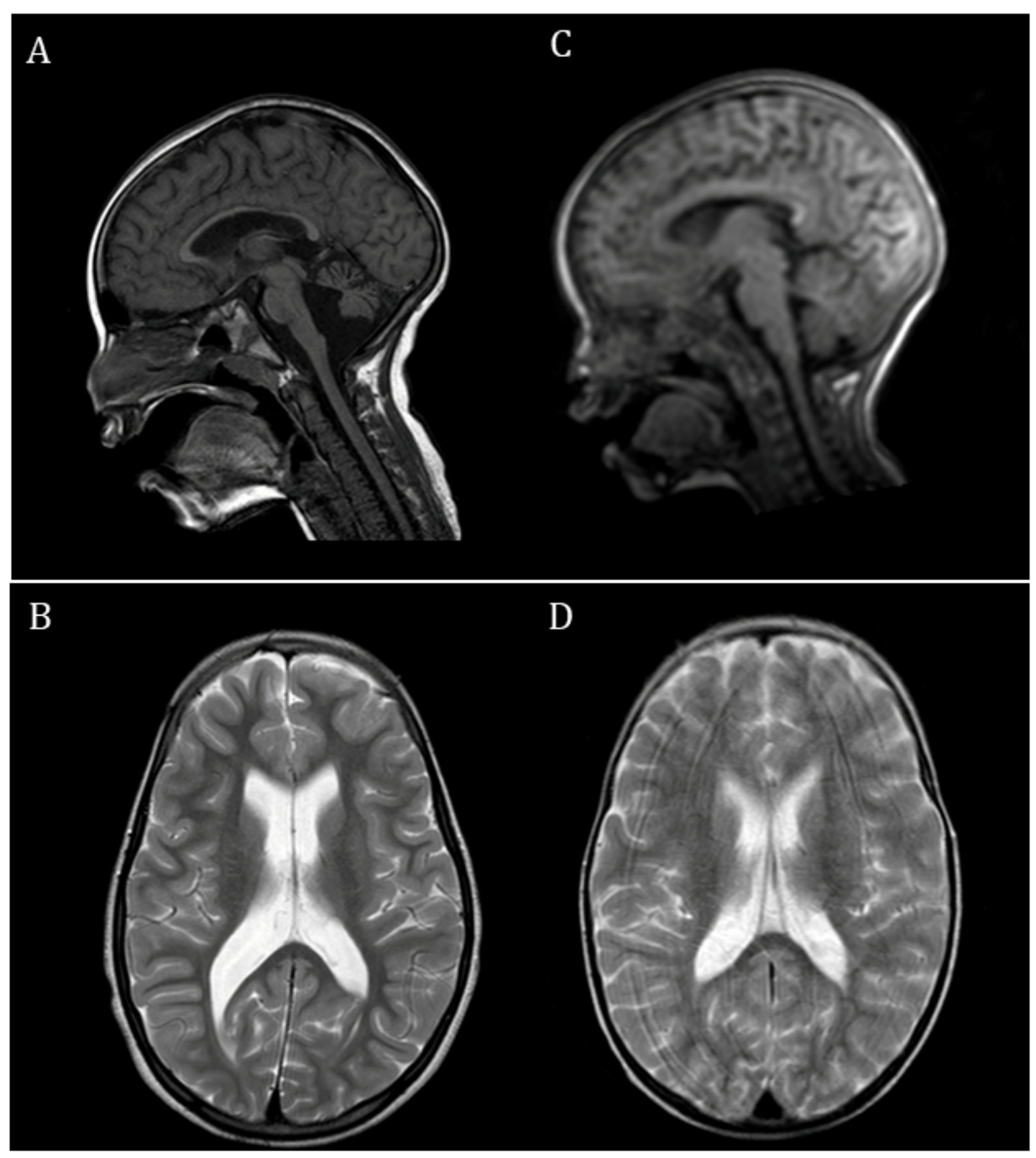

